\title{
The Rhizobium etli argC Gene Is Essential for Arginine Biosynthesis and Nodulation of Phaseolus vulgaris
}

\author{
Simona Ferraioli, Rosarita Taté, Emilia Caputo, Alessandro Lamberti, Anna Riccio, and \\ Eduardo J. Patriarca \\ International Institute of Genetics and Biophysics, C.N.R., Via G. Marconi 10, 80125 Naples, Italy \\ Accepted 11 October 2000.
}

\begin{abstract}
A Tn5-induced mutant strain (CTNUX5) of Rhizobium etli unable to grow with ammonium as the sole nitrogen source was isolated and characterized. Sequence analysis showed that $\mathrm{Tn} 5$ is inserted into an $\arg C$-homologous gene. Unlike its wild-type parent (strain CE3), the mutant strain CTNUX5 had an absolute dependency on arginine to grow. The $\arg C$ gene was cloned from the wild-type strain CE3, and the resulting plasmid, pAR207, after transformation was shown to relieve the arginine auxotrophy of strain CTNUX5. Unlike strain CE3 or CTNUX5pAR207, strain CTNUX5 showed undetectable levels of $\mathrm{N}$ acetyl- $\gamma$-glutamylphosphate reductase activity. Unless arginine was added to the growth medium, strain CTNUX5 was unable to produce flavonoid-inducible lipo-chitin oligosaccharides (nodulation factors) and to induce nodules or nodulelike structures on the roots of Phaseolus vulgaris.
\end{abstract}

Additional keywords: polyamines biosynthesis, root exudates.

The soil bacterium Rhizobium etli is able to induce the formation of nitrogen-fixing nodules on the root of common bean (Phaseolus vulgaris). From the point of view of the bacterial partner, the process of nodule formation involves bacterial multiplication in the rhizosphere, the recognition and infection of the root hairs, bacterial growth inside a network of intercellular infection threads, and the release of bacteria into the cytoplasm of plant cells. Within the invaded host cells the bacteria differentiate into symbiosomes that are able to reduce atmospheric dinitrogen into ammonia (Cermola et al. 2000; Tatè et al. 1994; Van Rhijn and Vanderleyden 1995).

Some metabolites and signals exchanged during symbiosis by both partners have been elucidated, but it is unclear which sources (carbon, sulfur, or nitrogen) the bacteria require or have available during their growth in the rhizosphere, inside the infection threads, and during symbiosome differentiation

Corresponding author: E. J. Patriarca, Telephone: +39 081 7257223; Fax: +39 081 7257319; E-mail: patriarca@iigbna.iigb.na.cnr.it

Nucleotide and/or amino acids sequence data are submitted to the EMBL, Genbank, and DDBJ sequence databases as accession no. AJ297961. inside the infected host cells. It was demonstrated, however, that various metabolic pathways of $R$. etli are important for an efficient symbiotic interaction (Noel et al. 1988; Tatè et al. 1997, Tatè et al. 1999a; Tatè et al. 1999b). With the exception of the methionine-requiring strain CTNUX23 (Tatè et al. 1999b), all strains of $R$. etli defective in amino acid biosynthesis, so far characterized, were able to induce nodules when tested in the absence of their nutritional requirement. Some strains, including CTNUX8-Cys-, CTNUX13-Ilv/Val- ${ }^{-}$, and CTNUX10-Asp/Asn', elicited normal nodules, whereas the effectiveness of nodules elicited by other mutants such as CTNUX4-Trp-, CTNUX36-Leu', and CTNUX20-His ${ }^{-}$is strongly reduced (Tatè et al. 1997, Tatè et al. 1999a; our unpublished data).

The ability of the amino acid-requiring mutants of $R$. etli to induce nodules and invade developing nodules may be explained if the bacteria acquire amino acids from the root cells. It was determined previously that some amino acids are released by the main root of a pea seedling (Van Egeraat 1975), but a clear-cut correlation between the amino acid composition of root exudates and the symbiotic phenotype of the amino acid-requiring mutants was never established. Preliminary experiments showed the presence of amino acids but not arginine in root exudates of common bean seedlings. Thus, an arginine-requiring mutant strain of $R$. etli, CTNUX5, was isolated and characterized.

To isolate mutants, the suicide plasmid pSUP1011 (Simon et al. 1983) was used to deliver Tn5 to the $R$. etli wild-type strain CE3, as previously described (Tatè et al. 1997). Mutants showing arginine-dependent growth were identified by simultaneous screening on plates (1.5\% agar) of ammoniumcontaining minimal medium (RMM) (Hooykaas et al. 1977), with or without $20 \mu \mathrm{g}$ of added arginine per ml. Two strains able to grow only in the presence of arginine (CTNUX5 and CTNUX14) were recovered from 1,500 Tn5 recipients.

Probing the $5 \mu \mathrm{g}$ of the total genomic DNA of strains CTNUX5 and CTNUX14 with a labeled Tn5 DNA fragment (5.5-kb HpaI fragment) established that in both strains the insertion was located on a single EcoRI fragment. Moreover, when the genomic DNAs were digested with BamHI, two fragments hybridized (data not shown). Therefore, each strain carried only one $\operatorname{Tn} 5$, which was located in a very closely 
linked genomic site. Because both strains also showed identical growth and symbiotic phenotypes, only one (strain CTNUX5) was chosen for further characterization. By selecting kanamycin-resistant transformants of Escherichia coli strain DH5 $\alpha$ (Woodcock et al. 1989), plasmid pAR174 carrying a portion of the $\operatorname{Tn} 5$ (including the kanamycin resistance cassette) plus the adjacent genomic DNA was isolated from strain CTNUX5 (Fig. 1A). The DNA sequence of the flanking DNA region, including that of the insertion point, was established with the Tn5out oligonucleotide. Sequence analysis revealed an open reading frame (ORF) (amino acids [aa] 216 to 308 of the deduced peptide sequence shown in Fig. 1C) that was similar to those of numerous prokaryotic and eukaryotic $\mathrm{N}$-acetylglutamylphosphate reductases (the $\arg C$ gene product), which are involved in the biosynthesis of arginine.
Southern hybridization of various restriction digests of DNA obtained from the wild-type strain of $R$. etli CE3 probed against a DNA fragment from pAR191 (Fig. 1A) yielded at least one strongly hybridizing band for each digest. The HindIII-digested DNA fragments, ranging from 5.2 to $5.7 \mathrm{~kb}$, were chosen to construct an enrichment library with plasmid pMP220 (Spaink et al. 1987) as a vector. The ligation mixture was used to transform competent cells of strain CTNUX5 (by electroporation), and the resulting cells were plated on RMM agar without arginine. From one of the transformants obtained, plasmid pAR207 carrying a 5.5-kb HindIII DNA fragment was isolated. We used the appropriate synthetic oligonucleotide primers to obtain the DNA sequence. A sequence analysis revealed one major ORF of $927 \mathrm{bp}$ and the predicted protein (Fig. 1C) 308 aa, shares 50\% of identical residues

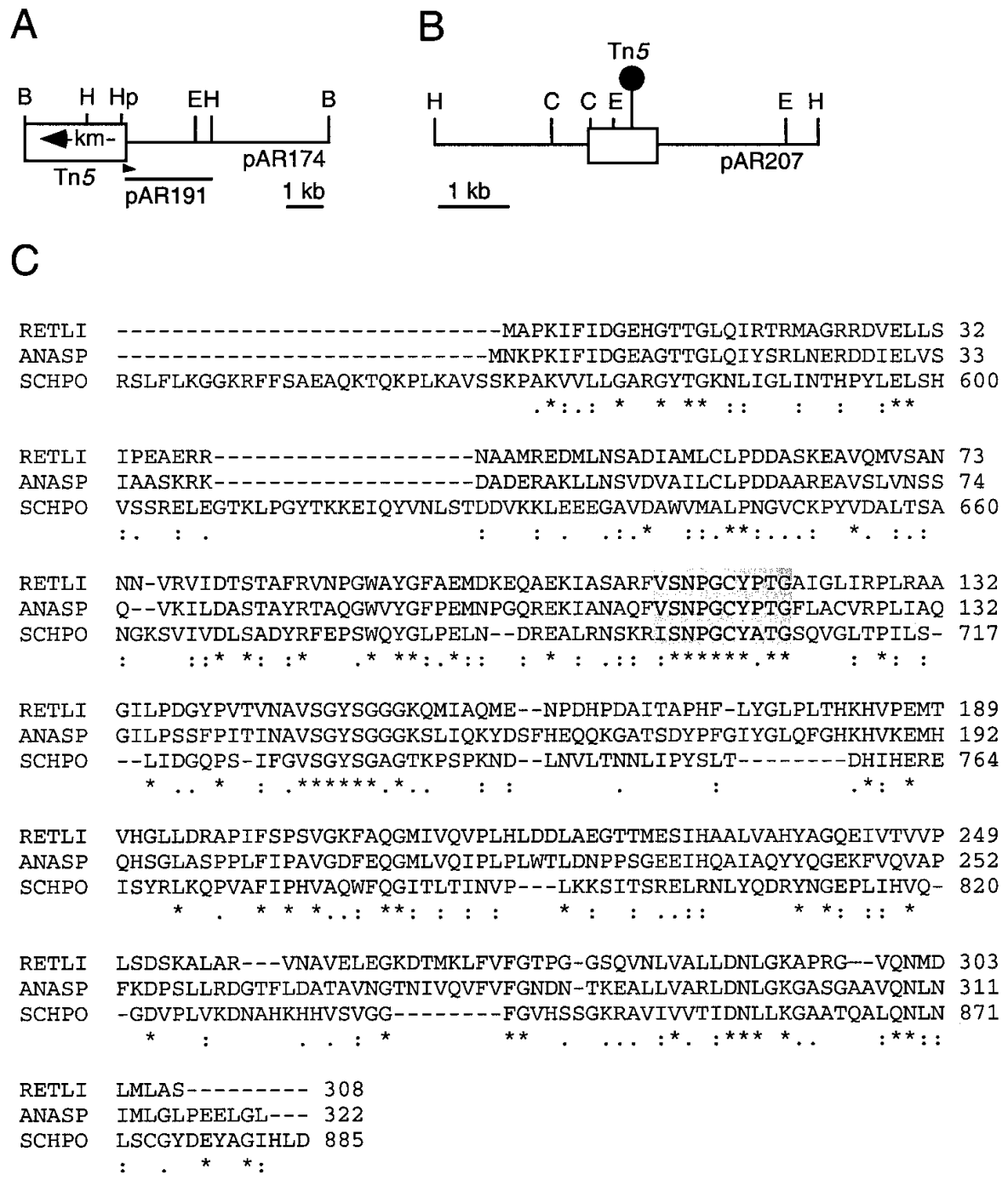

Fig. 1. A, Strategy to clone the Tn5 DNA flanking region from strain CTNUX5 of Rhizobium etli. A partial restriction map of the Tn5 DNA is shown. The black bar indicates the DNA fragment used as a probe to identify and clone the corresponding DNA region from the wild-type strain CE3. The Tn5outward reading primer is indicated by the arrowhead. B, Physical map of the $R$. etli DNA cloned into pAR207 with a partial restriction map. The boxed area in pAR207 represents the translated sequence of the $\arg C$ gene. The position of the Tn 5 insertion in the mutant strain CTNUX5 of $R$. etli is indicated. Restriction site positions are: $\mathrm{B}=$ BamHI; $\mathrm{C}=\mathrm{ClaI}$; $\mathrm{E}=$ EcoRI; $\mathrm{H}=$ HindIII; Hp = HpaI. C, Multiple sequence alignment is between the deduced peptides encoded by the $\arg C$ gene of Rhizobium etli (RETLI), the $\operatorname{argC}$ gene of Anabaena sp. (ANASP), and the C-terminal portion of the AR56 gene of Schizosaccharomyces pombe (SCHPO). Identical amino acid residues $(\star)$ and similarity $(\bullet)$ or conservative substitutions (:) between sequences are indicated. The amino acid sequences that fit with the active site of $N$-acetyl- $\gamma$-glutamylphosphate reductase enzymes are boxed. These sequence data are deposited in the EMBL, GenBank, and DDBJ data libraries under accession no. AJ297961. 
(68\% of similar residues over a 304-aa overlap) with the product of the Anabaena sp. $\operatorname{argC}$ gene (accession no. P54894) and $28 \%$ of identical residues over a 295-aa overlap with the C-terminal part (from position 567 to 850) of the product of the Schizosaccharomyces pombe AR56 gene (accession no. P31318). In yeast, $N$-acetylglutamate is converted into $N$-acetyl- $\gamma$-glutamylphosphate by a single multifunctional protein, the AR56 gene product, whereas in enteric bacteria, the same conversion requires the product of two genes, $\arg B$ and $\arg C$. Thus it was assumed that an $\arg C$ homologous gene is present in $R$. etli. The length of the $R$. etli $\arg C$ gene (308 aa, $32.9 \mathrm{kDa}$ ) is similar to those of other bacteria ( 35 to $38 \mathrm{kDa})$. One of the most conserved regions with respect to other reported $\mathrm{ArgC}$ proteins was the region around cysteine-117 (VSNPGCYPTG) (Fig. 1C), which is the active site of $N$-acetyl- $\gamma$-glutamylphosphate reductase (PROSITE database no. PDOC00941). Therefore, in strain CTNUX5 the gene interrupted by the Tn 5 most probably encodes the $\arg C$ gene product of bacteria. Upstream of $\arg C$ (up to $300 \mathrm{bp}$ ), a sequence analysis revealed the presence of a truncated ORF (89 codons). The predicted protein shares significant sequence similarity (73\% similar residues over a 61-aa overlap) with the C-terminal part of the agmatine ureohydrolase enzyme (encoded by the speB gene) of E. coli. In prokaryotes, this enzyme participates in polyamines biosynthesis, catalyzing the hydrolysis of agmatine into putrescine and urea (Szumanski and Boyle 1990). The putative stop codon (TGA) of this ORF is situated $61 \mathrm{bp}$ upstream of the putative start codon ATG of $\arg C$. In contrast, downstream of $\arg C$ (up to $300 \mathrm{bp}$ ) searches for sequence similarity (performed with BLAST software, National Center for Biotechnology-U. S. National Institutes of Health) gave no significant results.

To test the nutritional requirements of $R$. etli strains, the chemically defined $\mathrm{RMM}$ containing $5 \mathrm{mM} \mathrm{NH}_{4} \mathrm{Cl}$ and $1 \%$ glucose as nitrogen and carbon sources, respectively, was used. Strain CTNUX5 could grow in the arginine-containing medium but could not grow in the same medium supplemented with any other amino acid. Strain CTNUX5 was further characterized by testing its growth on RMM agar containing the compounds known as intermediates of the arginine biosynthesis. Strain CTNUX5 could grow in RMM containing $20 \mu \mathrm{g}$ of either acetylornithine, ornithine, or citrulline per $\mathrm{ml}$ but could not grow in the presence of acetylglutamate or arginininosuccinate. It is important to note that the wild-type strain of $R$. etli, CE3, could not grow with up to $200 \mu \mathrm{g}$ of argininosuccinate per $\mathrm{ml}$ as the sole nitrogen source, whereas it could grow with all the other intermediates tested, suggesting that $R$. etli is impermeable to argininosuccinate. Taking into consideration the arginine biosynthetic pathway of enteric bacteria, the nutritional requirements of strain CTNUX5 correspond to that of a mutant unable to convert $\mathrm{N}$-acetylglutamate into $\mathrm{N}$ acetylornithine (Glansdorff 1986). To test the ability of the wild-type locus to complement the growth phenotype, either plasmid pMP220 (vector alone) or pAR207 (Fig. 1B) was introduced into $R$. etli CTNUX5 by electroporation. Transformants bearing pAR207 (CTNUX5-pAR207) could grow in the absence of arginine, whereas those bearing pMP220 (CTNUX5-pMP220) could not. Therefore, the DNA fragment carried by pAR207 contains the information necessary to complement the Tn5 mutation.
To obtain more information about the function of $R$. etli ArgC, measurements of $N$-acetyl- $\gamma$-glutamylphosphate reductase activity were performed in different strains of $R$. etli. Bacteria grown in TYR medium (Tatè et al. 1999b) were harvested by low-speed centrifugation $(2,500 \times g)$, washed, and resuspended in ammonium-containing RMM. Cells were maintained for $3 \mathrm{~h}$ with shaking at $30^{\circ} \mathrm{C}$, harvested by highspeed centrifugation $(8000 \times g)$, and disrupted by sonic oscillation. The resulting crude extracts were used as a source for $N$-acetyl- $\gamma$-glutamylphosphate reductase activity, and the disappearance of NADPH (in the presence of $N$-acetylglutamate and ATP) was measured spectrophotometrically. The rates were corrected for consumption of NADPH measured in the absence of added $N$-acetylglutamate. The $N$-acetyl- $\gamma$ glutamylphosphate reductase activity measured with the wildtype strain CE3 was $0.25 \mu \mathrm{mol} \mathrm{min} \mathrm{mg}^{-1}$, the activity of the CTNUX5 mutant was approximately $0.005 \mu \mathrm{mol} \mathrm{min} \mathrm{mg}^{-1} \mathrm{mg}^{-1}$, and the activity of strain CTNUX5-pAR207 was $0.82 \mu \mathrm{mol}$ $\mathrm{min}^{-1} \mathrm{mg}^{-1}$. Therefore, in $R$. etli the $\arg C$ gene product is essential for the NADPH-dependent reduction of $N$-acetyl- $\gamma$ glutamylphosphate.

To test the capacity of the $R$. etli strain CTNUX5 ( $\arg C:: \operatorname{Tn} 5)$ to nodulate $P$. vulgaris, seeds were surfacesterilized (immersed in 5\% hydrogen peroxide for $30 \mathrm{~min}$ and washed twice in sterile water) and germinated at $23^{\circ} \mathrm{C}$ on a petri dish containing $1 \%$ water agar. Roots of germinated seeds were inoculated with $R$. etli cells suspended in the inoculation buffer (50 $\mathrm{mM}$ phosphate buffer, $\mathrm{pH} 7.0)$ at a required density (approximately $10^{5} \mathrm{ml}^{-1}$ ). Two weeks after inoculation, the presence of nodules on the roots of plants inoculated with strain CE3 and the absence of nodules (or nodulelike structures) on the roots inoculated with strain CTNUX5 was observed, indicating that strain CTNUX5 was unable to induce nodulation on common beans. Moreover, at different times after inoculation the roots were harvested, fixed, stained with methylene blue, and observed by light microscopy (Fig. 2), as previously described (Tatè et al. 1994). The early root responses such as root-hair curling, mitotic

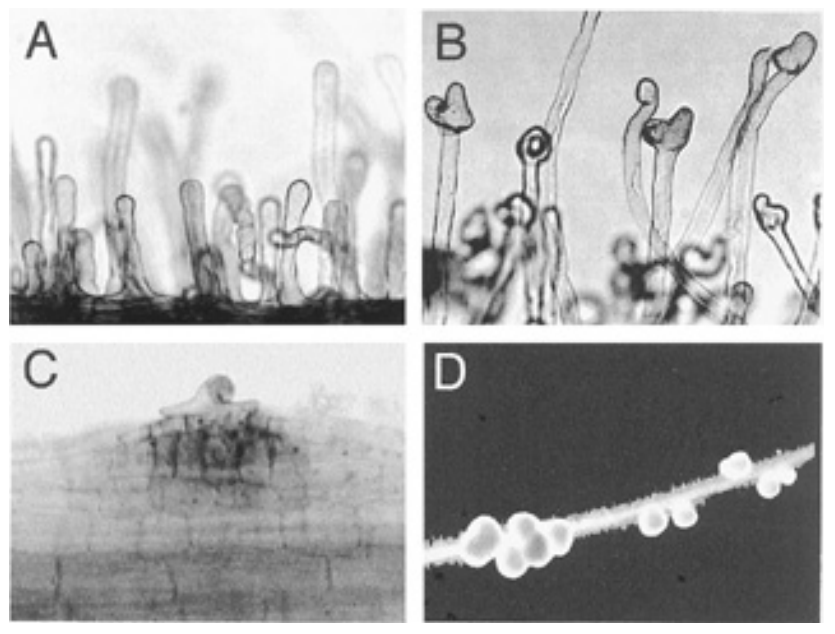

Fig. 2. The response of Phaseolus vulgaris roots after inoculation with $\mathbf{A}$, the $\arg C$ mutant strain CTNUX5 ( $24 \mathrm{~h}$ postinoculation); and $\mathbf{B}$ and C, strain CTNUX5 in the presence of arginine (24 and $96 \mathrm{~h}$ postinoculation, respectively). D, Twelve-day-old nodules induced by strain CTNUX5-pAR207 of Rhizobium etli. 
activity of root cells (from the outer cortex), and formation of infection threads were observed with strain CE3 but not with strain CTNUX5 (Fig. 2A). To test the effect of the addition of arginine on the nodulation ability of the $\arg C$ mutant strain CTNUX5, nodulation experiments were performed with the same nitrogen-free medium (Jensen 1942) but supplemented with $50 \mu \mathrm{g}$ of arginine (the nutritional requirement of the auxotrophic mutant) per ml. Under these conditions, mutant strain CTNUX5 was able to induce, although less efficiently, the same root reactions (i.e., root hair curling, induction of mitotic activity, and formation of infection threads) induced by the wild-type strain CE3 (Fig. 2B and C). Finally, strain CTNUX5-pAR207 induced nodules (Fig. 2D), although with a lower nitrogen-fixation capacity $(65 \%$, measured as the acetylene reduction activity) than the nodules induced by the parent strain CE3. These data indicate that the DNA fragment carried by pAR207 was able to rescue the loss of nodulation ability of strain CTNUX5. However, because strain CTNUX5-pAR207 showed a higher (threefold) level of $\mathrm{N}$ acetyl- $\gamma$-glutamylphosphate reductase activity, it is possible that alteration in the intracellular level of arginine (or a metabolic precursor-product) may have a detrimental effect on the symbiotic interaction. Otherwise, plasmid pAR207 may be lost during nodule invasion because the bacteria divide inside the plant cells in the absence of selective pressure.

To test the ability of strain CTNUX5 to produce Nod factors, its growth medium was investigated for the presence of flavonoid-inducible lipo-chitin oligosaccharides by radiolabeling the cells with $\mathrm{D}-\left[1-{ }^{14} \mathrm{C}\right]$-glucosamine (Fig. 3). Bacteria were grown overnight at $30^{\circ} \mathrm{C}$ (from an initial optical density value at 590 of 0.05 ) in $1 \mathrm{ml}$ of RMM minimal medium containing $1 \mu \mathrm{Ci}$ of $\mathrm{D}-\left[1-{ }^{14} \mathrm{C}\right]$-glucosamine. Nitrate and mannitol were used as nitrogen and carbon sources, respectively. Two micromolars of naringenin was used as the inducer of the

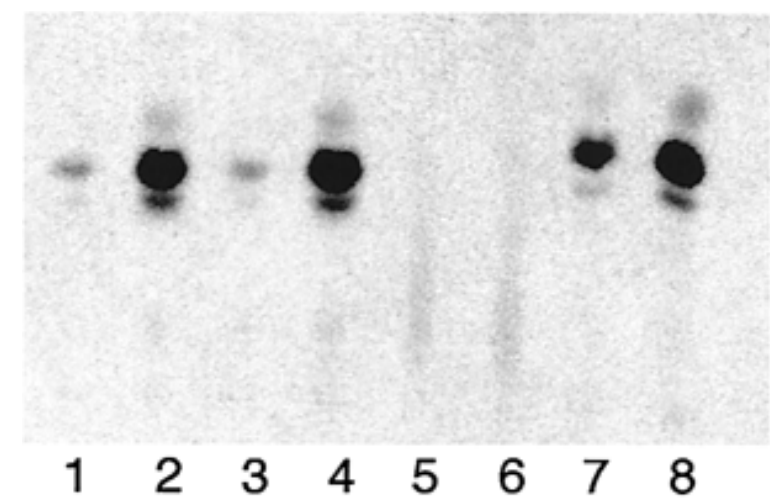

Fig. 3. Thin-layer chromatographic analysis of radiolabeled compounds produced by Rhizobium etli. Lanes 1 to 4: strain CE3 (wild-type); lanes 5 to 8: strain CTNUX5 ( $\left.\operatorname{argC}^{-}\right)$. Bacteria were incubated in the presence of $2 \mu \mathrm{M}$ naringenin (lanes $2,4,6$, and 8 ) or in the absence of the inducer $(1,3,5$, and 7$)$. The cultures were also supplemented with $50 \mu \mathrm{g}$ of arginine per $\mathrm{ml}$ (lanes 3, 4, 7, and 8). One milliliter of culture medium was extracted with $0.5 \mathrm{ml}$ of water-saturated $n$-butanol, which was removed by evaporation, and the dry pellet was resuspended in $20 \mu \mathrm{l}$ of $n$ butanol. Three microliters of samples were loaded on a reverse-phase $\mathrm{C}_{18}$-coated silica $100 \%$ octyldecyl silane plate (Sigma, Milan, Italy) with acetonitrile and water $(1: 1, \mathrm{vol} / \mathrm{vol})$ as a solvent. The plate was dried, and the radiolabeled compounds were visualized with a PhosphorImager gel scanner (Molecular Dynamics, Sunnyvale, CA, U.S.A.) after 24 h of exposure. nodulation genes. The radiolabeled compounds were extracted and analyzed by thin-layer chromatography, essentially as described previously (Spaink et al. 1992). In the presence of the inducer, the wild-type strain CE3, which was used as a control, produced two main radiolabeled spots (Fig. 3, lane 2). As previously observed (Cardenas et al. 1995, Tatè et al. 1999a; Tatè et al. 1999b), metabolites that showed similar chromatographic behavior also were detected in the uninduced culture (Fig. 3, lane 1), although at lower levels. Moreover, the addition of $50 \mu \mathrm{g}$ of arginine per $\mathrm{ml}$ to the growth medium of strain CE3 showed no effect on the production of flavonoid-inducible compounds. In contrast, strain CTNUX5 was unable to grow and produce radiolabeled compounds, either in the presence or absence of naringenin (Fig. 3, lanes 5 and 6), unless arginine was added to the growth medium (Fig. 3 , lanes 7 and 8). In the presence of arginine and the absence of the naringenin (uninduced cells), mutant strain CTNUX5 produced a significantly higher level of radiolabeled compounds (showing a similar chromatographic behavior) than wild-type strain CE3 (Fig. 3, lanes 3 and 7). Thus in strain CTNUX5, the production of Nod factors appears to be partially induced (or derepressed) in the absence of naringenin. Interestingly, it was observed previously (Tatè et al. 1999a; Tatè et al. 1999b) that higher amounts of Nod factors are produced by strains CTNUX4-Trp ${ }^{-}$and CTNUX23-Met ${ }^{-}$when the auxotrophy is complemented with the corresponding amino acid (in the absence of naringenin).

We propose that in root exudates of germinated seedlings the arginine concentration, if present, is too low because under free-living conditions the $\arg C$ gene is essential to allow the growth of $R$. etli in minimal medium. The capacity of strain CTNUX5 to induce cortical cell division (Fig. 2) and produce Nod factors (Fig. 3) could be restored by the addition of $50 \mu \mathrm{g}$ of arginine per $\mathrm{ml}$ to the medium. Therefore, it appears that a shortage of arginine, rather than the absence of the ArgC protein per se, is responsible for the defective nodulation phenotype of mutant strain CTNUX5. It was observed earlier that mutants of $R$. meliloti and $R$. leguminosarum with defects in arginine biosynthesis formed nodules $\left(\mathrm{Nod}^{+}\right)$on the roots of alfalfa or pea, respectively (Kerppola and Kahn 1988; Pain 1979). The R. meliloti mutants blocked in the latter part of the arginine biosynthetic pathway elicited effective (i.e., able to fix nitrogen) nodules (Kerppola and Kahn 1988). In contrast, mutants blocked in the early steps of the pathway formed ineffective nodules (Kerppola and Kahn 1988). However, unlike strain CTNUX5 of $R$. etli, $\mathrm{Arg}^{-}$mutants of other Rhizobium species were able to induce the formation of nodules, thus indicating that they are able to produce and release active Nod factors. Moreover, because root and nodule metabolisms are different for different legumes (Schubert 1986), it is possible to speculate that the bacterial nutrients are specific for each symbiotic relationship.

Strain CTNUX5 ( $\left.\mathrm{Arg}^{-}\right)$is the second amino acid-requiring mutant strain of $R$. etli unable to induce nodules on the root of $P$. vulgaris. In fact, it was reported previously that a methionine (Met) $)^{-}$(metZ) strain of $R$. etli, CTNUX23, also is unable to induce nodules (Tatè et al. 1999b). Methionine and arginine are metabolic precursors in the biosynthetic pathway of polyamines such as agmatine, putrescine, spermidine, and spermine. Finally, it was shown previously that the ability of the Uromyces phaseoli fungus to infect $P$. vulgaris depends on 
its ability to produce polyamines (Venkat Rajam et al. 1985). The involvement of polyamines in the nodulation ability of $R$. etli has not yet been investigated.

\section{ACKNOWLEDGMENTS}

This work was supported by a grant from EEC (EURATINE: BIOTEC94-2310). We thank C. Rallo and M. Valenzi for computer analysis, C. Sole for technical assistance, and O. Piedimonte for help with the figures.

\section{LITERATURE CITED}

Cardenas, L., Dominguez, J., Quinto, C., Lopez-Lara, I. M., Lugtenberg, B. J. J., Spaink, H. P., Rademarker, G. J., Haverkamp, J., and ThomasOates, J. E. 1995. Isolation, chemical structures and biological activity of lipo-chitin oligosaccharide nodulation signals from Rhizobium etli. Plant Mol. Biol. 29:453-464.

Cermola, M., Fedorova, E., Taté, R., Riccio, A., Favre, R., and Patriarca, E. J. 2000. Nodule invasion and symbiosome differentiation during Rhizobium etli-Phaseolus vulgaris symbiosis. Mol. Plant-Microbe Interact. 13:733-741.

Glansdorff, N. 1986. Biosynthesis of arginine and polyamines. Pages 321-344 in: Escherichia coli and Salmonella typhimurium: Cellular and Molecular Biology, Vol. 1. F. C. Neidhardt, ed. American Society for Microbiology, Washington.

Hooykaas, P. J. J., Clapwijk, P. M., Nuti, M. P., Shilperoort, R. A., and Roersch, A. 1977. Transfer of the Agrobacterium tumefaciens Ti plasmid to avirulent Agrobacteria and to Rhizobium ex-planta. J. Gen. Microbiol. 98:477-484.

Jensen, H. L. 1942. Nitrogen fixation in leguminous plants. General characters of root-nodule bacteria isolated from species of Medicago and Trifolium in Australia. Proc. Linn. Soc. NSW 66:98-108.

Kerppola, T. K., and Kahn, M. L. 1988. Symbiotic phenotypes of auxotrophic mutants of Rhizobium meliloti 104A14. J. Gen. Microbiol. 134:913-919.

Noel, K. D., Diebold, R. J., Cava, J. R., and Brink, B. A. 1988. Rhizobial purine and pyrimidine auxotrophs: Nutrient supplementation, genetic analysis, and the symbiotic requirement for the novo purine biosynthesis. Arch. Microbiol. 149:499-506.

Pain, A. N. 1979. Symbiotic proprieties of antibiotic-resistant and auxotrophic mutants of Rhizobium leguminosarum. J. Appl. Bacteriol.
47:53-64.

Schubert, K. R. 1986. Products of biological nitrogen fixation in higher plants: synthesis transport and metabolism. Annu. Rev. Plant Physiol. 37:539-574.

Simon, R., Priefer, U., and Puhler, A. 1983. A broad host range mobilization system for in vivo genetic engineering: Transposon mutagenesis in gram-negative bacteria. Biotechnology 1:784-791.

Spaink, H. P., Okker, R. J. H., Wijffelman, C. A., Pees, E., and Lugtenberg, B. J. J. 1987. Promoters in the nodulation region of the Rhizobium leguminosarum Sym plasmid pRL1JI. Plant Mol. Biol. 9:27-39.

Spaink, H. P., Aarts, A., Stacey, G., Bloemberg, G. V., Lugtenberg, B. J. J., and Kennedy, E. P. 1992. Detection and separation of Rhizobium and Bradyrhizobium Nod metabolites using thin-layer chromatography. Mol. Plant-Microbe Interact. 5:72-80.

Szumanski, M. B. W., and Boyle, S. M. 1990. Analysis and sequence of the speB gene encoding agmatine ureohydrolase, a putrescine biosynthetic enzyme in Escherichia coli. J. Bacteriol. 172:538-547.

Taté, R., Patriarca, E. J., Riccio, A., Defez, R., and Iaccarino, M. 1994 Development of Phaseolus vulgaris root nodules. Mol. Plant-Microbe Interact. 7:582-589.

Taté, R., Riccio, A., Iaccarino, M., and Patriarca, E. J. 1997. A cysG mutant strain of $R$. etli pleiotropically defective in sulfate and nitrate assimilation. J. Bacteriol. 179:7343-7350.

Taté, R., Riccio, A., Caputo, E., Cermola, M., Favre, R., and Patriarca, E. J. 1999a. The Rhizobium etli trpB gene is essential for an effective symbiotic interaction with Phaseolus vulgaris. Mol. Plant-Microbe Interact. 12:926-933.

Taté, R., Riccio, A., Caputo, E., Iaccarino, M., and Patriarca, E. J. 1999b. The Rhizobium etli metZ gene is essential for methionine biosynthesis and nodulation of Phaseolus vulgaris. Mol. Plant-Microbe Interact. 12:24-34.

Van Egeraat, A. W. 1975. Exudation of ninhydrin-positive compounds by pea seedling roots: A study of the sites of exudation and of the composition of the exudate. Plant Soil 42:15-36.

Van Rhijn, P., and Vanderleyden, J. 1995. The Rhizobium-plant symbiosis. Microbiol. Rev. 59:124-142.

Venkat Rajam, M., Weinstein, L. H., and Galston, A. W. 1985. Prevention of a plant disease by specific inhibition of fungal polyamine biosynthesis. Proc. Natl. Acad. Sci. 82:6874-6878.

Woodcock, D. M., Crowther, P. J., Doherty, J., Jefferson, S., DeCruz, E., Noyer-Weidner, M., Smith, S. S., Michael, M. Z., and Graham, M. W. 1989. Quantitative evaluation of Escherichia coli host strains for tolerance to cytosine methylation in plasmid and phage recombinants. Nucleic Acid Res. 17:3469-3478. 